\title{
Rapid Label-free Nanotechnological Immunoassay for Analysis of Candidate Malaria Vaccines
}

\author{
Giulio Brunetti, Annalisa De Pastina and Martin Hegner* \\ Centre for Research on Adaptive Nanostructures and Nanodevices (CRANN), School of Physics, Trinity College Dublin, \\ Dublin, Ireland
}

*Corresponding author: Martin Hegner, Centre for Research on Adaptive Nanostructures and Nanodevices (CRANN), School of Physics, Trinity College Dublin, Dublin, Ireland, E-mail: hegnerm@tcd.ie

We recently demonstrated a direct single-step label-free quantitative immunoassay in serum, investigating malaria vaccines [1]. Our method exhibits 1 pg mass sensitivity at concentrations of approx. $100 \mathrm{pg} / \mathrm{ml}$, on par with the goldstandard multi-step enzyme-linked immunosorbent assay (ELISA). The nanomechanical assays perform faster and enable simultaneous mass uptake studies of multiple targets, due to epitope-specific recognition [1]. In nanomechanical assays parallel measurement points are represented over time ( $5 \mathrm{~s}$ time resolution), thus providing additional temporal information compared with the ELISA output of averaged single end-point measurements. This commentary elucidates the additional advantages of label-free differential assays over the classical immunosorbent assay.

\section{DIFFERENTIAL NANOMECHANICAL MEASUREMENTS COMPARISON TO GOLD- STANDARD ELISA}

\section{Protein physisorption in ELISA assays}

In most ELISA tests, the individual assays are designed by physisorbing receptor proteins or antibodies to non-treated polystyrene surfaces of microtiter plates through passive adsorption [2]. These plates are provided with different surface properties ranging in hydrophobicity and charge [3, 4]. Soluble proteins orient hydrophobic amino acids towards their core, while polar and charged amino acids face the surrounding solution and ions in order to maintain their folding and consequent 3D structure [5]. Soluble proteins facilitate binding to a hydrophobic surface by partially orienting their internal hydrophobic amino acids against the surface to become more hydrophobic. This way, the surface binding energy is maximized. However, hydrophobic interfaces may partially denature proteins that bind to them [6], therefore great care has to be taken to minimize this effect $[7,8]$.
The overall protein sequence and amino acid properties are crucial to anchor specific epitopes to the assay interfaces. Indeed, on high-binding hydrophobic ELISA plates [2], alanines within alpha helical structures will be oriented towards the polystyrene surface, which can hamper the correctly folded structure in protein loops.

\section{Protein immobilization in nanomechanical assays}

In contrast, on nanomechanical sensors the malaria vaccine candidates with the PE-peptidomimetics [9-11] were coupled via lysine's residuals or primary amines to a self-assembled monolayer (SAM) that becomes even more hydrophilic after conversion. The coupling was providing a pin-point covalent attachment $[7,12]$. Proteins that were bound this way retained their 3D folding better, since solutions and ions have threedimensional access. In more detail, from the commented study, UK39, exhibits several alanines that are more likely to be located inwards (away from $\mathrm{H}_{2} \mathrm{O}$ ) in the properly folded loop [10] and one primary amine for covalent coupling. AMA49-C1 exposes 4 lysines and one primary amine $[9,11]$ to allow covalent coupling to the NHS-activated dithiobis(1succinimidyl undecanoate (DSU) SAM on gold interfaces (see [1] Figure 1).

The binding of vesicle membranes integrated with delicate membrane receptors to DSU monolayers also proved favorably for the subsequent nanomechanical analysis [13]. The membrane proteins did not lose their integrity. Specific binding of T4 phages to the embedded FhuA receptors, anchored at their $\mathrm{NH}_{2}$ containing residues, could be demonstrated and also verified image via SEM imaging [13].

We would define this direct coupling to an amino reactive surface bound monolayer as an "active immobilization", in contrast to the passive immobilization on high-bind ELISA plates. Indeed, depending on how ELISA sandwich assays are 
Sensor Schematic

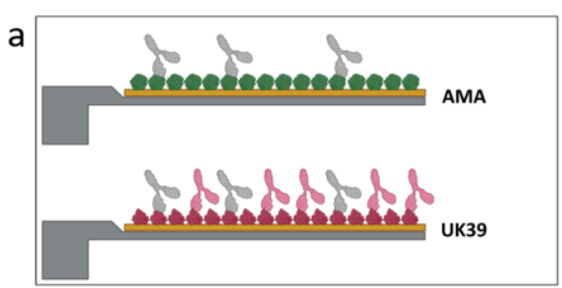

Frequency shift

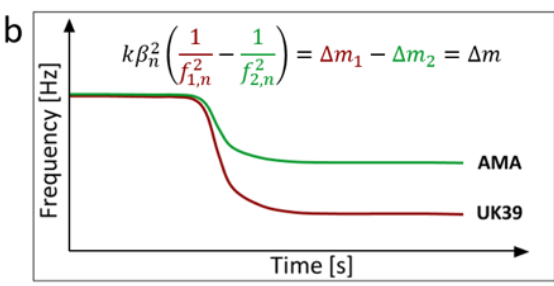

Differential mass (UK39 - AMA)

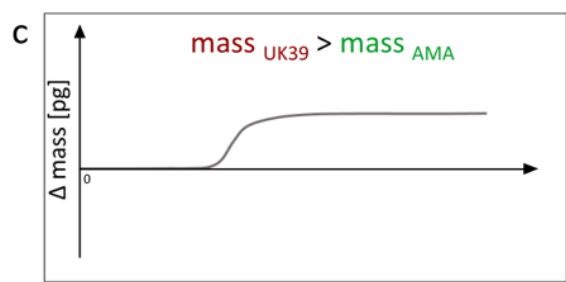

Figure 1 - Differential mass assay. Schematic of two silicon cantilevers functionalized with gold, self-assembled monolayers and AMA (green) or UK39 (red) peptides. The epitope-specific antibody recognition (panel a) (red antibodies towards UK39 and grey antibodies as unspecific interactions) generates a frequency shift based on the different sensor functionalizations (panel b). Taking the characteristics of the fluid around the sensors and the sensors' dimensions into account, the frequency shifts are converted into a mass addition towards one or the other epitope (panel c) [15, 17, 20, 22, 23].

designed, the proteins or antibodies that act as receptors for diagnostic targets, are passively bound to the surface.

\section{Differential nanomechanical analysis}

The micron-sized nanomechanical sensors are normally actuated at higher modes and their mechanics follows the harmonic oscillator model in a liquid environment [14]. The minimum detectable mass is given if one takes the minimum measurable frequency change. The sensors are exposed synchronously to the same sample and multiples of individual functionalizations [15] allow for averaging of the specific mechanical response. A minimum of two mechanically equivalent sensors is required [16], we currently use 18 in parallel [15]. Synchronised differential readout with in-situ reference probes is mandatory to avoid convolution with external environmental factors. Such differential analysis also eliminates thermal experimental drifts, flow-induced disturbances, and unspecific interactions as present in serum samples (see Figure 1) [1, 13, 15-23].

In an optimized nanomechanical differential assay, the chemical, topographic, polar, charged receptor interface of a sensor used as a reference, should mimic the actual molecular epitope as best as possible. Therefore, the assay should allow to specifically distinguish between the peptidomimetics PEUK39 and PE-AMA49-C1.

After the peptides are covalently bound to the sensor's functionalized DSU monolayers, the whole PE-amino acids vaccine candidates' epitopes are presented to the fluid. Differential readout allows to subtract all interactions that occur at the hydrophobic end or at the phosphatidyl-ethanolamine, so only the interactions at the peptide loops are measured as the purely peptide-specific recognition pattern.

When using human serum albumin (HSA) on the sensor surfaces as a reference, there are several additional epitopes available, apart from the targeted ones. In fact, HSA must denature slightly when being physisorbed, and can be bound to by different molecules in serum. Such detection on the surface has nothing to do with UK39. Even if a decrease in binding is noticed, as in the case of HSA passivation on ELISA surfaces, some recognition patterns can be attributed to serum proteins including primary and secondary immunoglobulins.

Since we are interested in the pure signal towards UK39 or AMA49-C1 in the malaria vaccine study, it is better to decorate the reference sensors of each assay with PE-UK39 (if one wants to measure AMA49-C1) or PE-AMA49-C1 (if one wants to measure UK39).

A schematic of the differential nanomechanical measurement is represented in Figure 1.

Comparing the assay result of UK39 - AMA49-C1 nanomechanically allows to directly measure the minute details of the antigenic epitopes themselves. This direct comparison of several targets in one well is not possible on an ELISA plate, where individual wells have distinct functionalizations, and where the remaining surfaces besides the antigen-receptor will be passivated as much as possible with HSA to avoid unspecific interactions. However, with only HSA as a reference, interactions that are not specifically related to the UK39 (or AMA49-C1) peptide can result. Once the first antibodies or antigens are bound, the secondary antibodies in the ELISA assay cannot differentiate whether primary layer antibodies are bound to UK39 or to a different epitope. This can lead to an overestimation or an underestimation of the actual signal and is normally optimized during the ELISA assay validation to minimize such effects.

The same applies to nanomechanical assays where, evaluating only an individual sensor, would also lead to an under- $/$ overestimation of specific interactions. This effect can only be minimized when the sensor surfaces in a measurement are directly compared $i n$-situ via multiple sensors. 
a

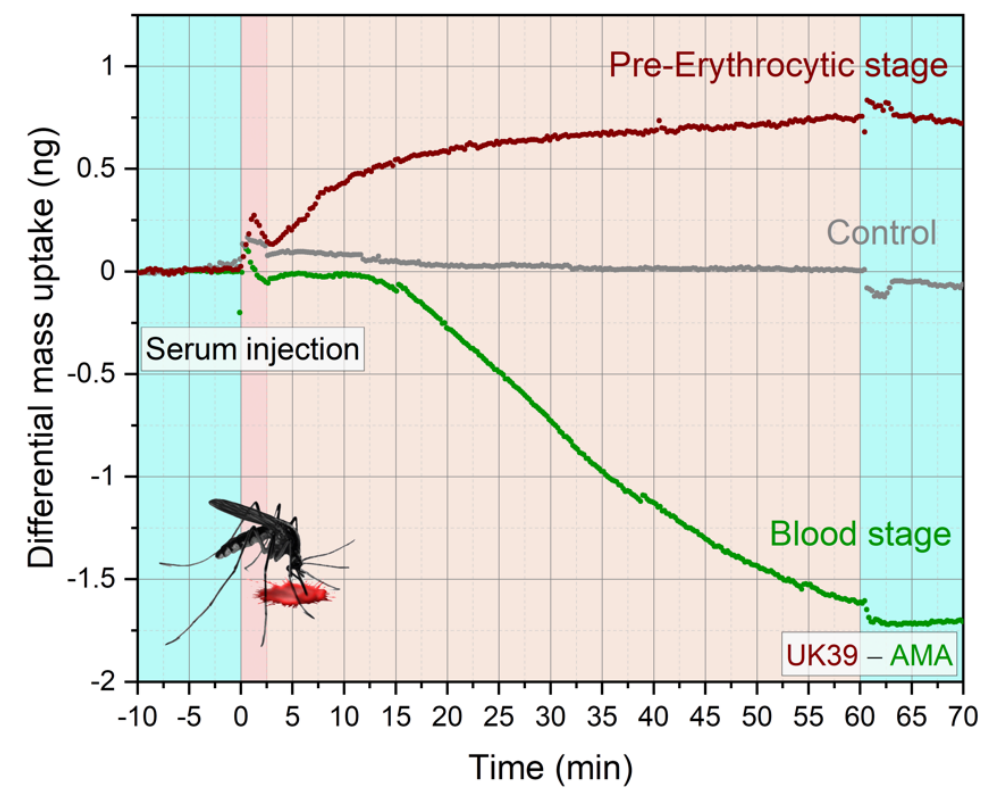

b

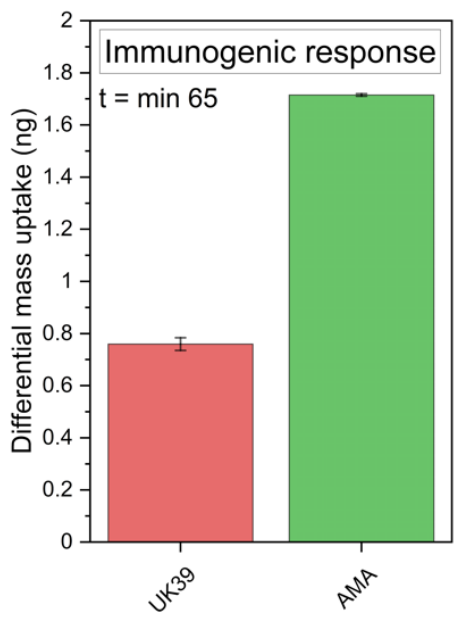

Figure 2 - Differential immunogenic serum response of adult volunteers' to malaria vaccine candidate (Adapted from [1]). a) Upon injection of the serum samples at time 0 minutes the sensor oscillations are slowing down due to specific binding of proportionally more molecules to one of the sensor types. Differential read-out allows direct discrimination between the two disease stages, the pre-erythrocytic stage induced by the vaccine candidate or the blood stage induced by the malaria mosquito bite (occasionally present in the non-naive volunteers). b) The bar graph represents the output of averaged single end-point measurements at 65 minutes, over a time frame of 5 minutes comparable to ELISA. The light blue background indicates stabilization in buffer (PBS), the dark pink represents serum sample injection, and the light pink denotes signal stabilization in serum (no flow). Differential analysis eliminates thermal drifts, flow-induced disturbance and unspecific interactions and will result in an absolute mass evaluation.

In nanomechanical assays we cannot exclude, by measuring masses bound in liquid on one sensor only, whether they are strictly related to the diagnostic target, to other unspecific proteins, or to variations in the hydrodynamic environment. Therefore, the contribution to the signal based on molecular recognition absolutely requires differential analysis. As mentioned above it is better to utilize receptor layers that are overall similar but differ slightly in the molecular recognition pattern. This is key to the resulting sensitive mass differences in UK39 - AMA49-C1 (AMA) measured in serum (see Figure 2). Furthermore, as mentioned earlier, all environmental effects (e.g. temperature or viscosity changes) are subtracted.

Label-free nanomechanical differential assays have been demonstrated over the last decades in the field of genomics [24-26], proteomics [1, 27, 28], microbiomics [29, 30] and hemostasis $[20,21]$. One should never turn a blind eye towards technological developments, whether these will be picked up as complementary technology and become mainstream is depending on many factors.

\section{CONFLICT OF INTEREST}

There is no conflict of interest to declare

\section{REFERENCES}

1. Brunetti G, Padovani F, De Pastina A, Rotella C, Monahan A, Hoffman SL, et al. Nanotechnological immunoassay for rapid label-free analysis of candidate malaria vaccines. Nanoscale. 2021;13(4):2338-49. Epub 2021/01/14. doi: 10.1039/d0nr08083g. PubMed PMID: 33438712.

2. Tan J, Sack BK, Oyen D, Zenklusen I, Piccoli L, Barbieri S, et al. A public antibody lineage that potently inhibits malaria infection through dual binding to the circumsporozoite protein. Nature Medicine. 2018;24:401.

3. Bergeron $\mathrm{AB}$, Bortz $\mathrm{CJ}$, Rossi A. Corning Medium and High Binding ELISA Microplates for Select Target Size Binding Assays 2019. Available from: https://www.corning.com/media/ worldwide/cls/documents/applications/CLS-AN-497\%20DL.pdf.

4. Esser P. Thermo Scientifc Solid Phase Guide 2019 [cited 2021]. Available from: https://assets.thermofisher.com/TFS-Assets/ LCD/Scientific-Resources/Thermo_Scientific_Solid_Phase_ Guide.pdf. 


\section{Commentary}

5. Dill KA, Ozkan SB, Shell MS, Weikl TR. The Protein Folding Problem. Annual Review of Biophysics. 2008;37(1):289-316. PubMed PMID: 18573083.

6. Thurow H, Geisen K. Stabilisation of dissolved proteins against denaturation at hydrophobic interfaces. Diabetologia. 1984;2(0012-186X (Print)):212-8.

7. Hoffmann AS, Hubbell JA. Surface immobilized Biomolecules. In: Ratner B, Hoffman A, Schoen F, Lemons J, editors. Biomaterials Science: An Introduction to Materials in Medicine. 2nd Edition. London, San Diego: Academic Press/Elsevier; 2004. p. 225 - 37.

8. Rathner B, Hoffman AS. Nonfouling surfaces. In: Ratner B, Hoffman A, Schoen F, Lemons J, editors. Biomaterials Science: An Introduction to Materials in Medicine. 2nd Edition. London, San Diego: Academic Press/Elsevier; 2004. p. 197 - 201.

9. Mueller MS, Renard A, Boato F, Vogel D, Naegeli M, Zurbriggen $\mathrm{R}$, et al. Induction of parasite growth-inhibitory antibodies by a virosomal formulation of a peptidomimetic of loop I from domain III of Plasmodium falciparum apical membrane antigen 1. Infection and immunity. 2003;71(8):4749-58. Epub 2003/07/23. PubMed PMID: 12874357; PubMed Central PMCID: PMCPMC166038.

10. Okitsu SL, Kienzl U, Moehle K, Silvie O, Peduzzi E, Mueller MS, et al. Structure-activity-based design of a synthetic malaria peptide eliciting sporozoite inhibitory antibodies in a virosomal formulation. Chemistry \& Biology. 2007;14(5):577-87.

11. Pfeiffer B, Peduzzi E, Moehle K, Zurbriggen R, Glück R, Pluschke G, et al. A Virosome-Mimotope Approach to Synthetic Vaccine Design and Optimization: Synthesis, Conformation, and Immune Recognition of a PotentialMalaria-VaccineCandidate. Angew Chem Int Ed. 2003;42:2368 - 71.

12. Wagner P, Hegner M, Kernen P, Zaugg F, Semenza G. Covalent immobilization of native biomolecules onto $\mathrm{Au}$ (111) via $\mathrm{N}$-hydroxysuccinimide ester functionalized self-assembled monolayers for scanning probe microscopy. Biophysical Journal. 1996;70(5):2052-66.

13. Braun T, Ghatkesar MK, Backmann N, Grange W, Boulanger $\mathrm{P}$, Letellier L, et al. Quantitative time-resolved measurement of membrane protein-ligand interactions using microcantilever array sensors. Nature Nanotechnology. 2009;4(3):179.

14. Eysden CAV, Sader JE. Frequency response of cantilever beams immersed in viscous fluids with applications to the atomic force microscope: Arbitrary mode order. Journal of Applied Physics. 2007;101(4):044908. doi: 10.1063/1.2654274.

15. De Pastina A, Padovani F, Brunetti G, Rotella C, Niosi F, Usov $\mathrm{V}$, et al. Multimodal real-time frequency tracking of cantilever arrays in liquid environment for biodetection: Comprehensive setup and performance analysis. Review of Scientific Instruments. 2021;92(6):065001. doi: 10.1063/5.0047631.

16. Lang HP, Hegner M, Gerber C. Cantilever array sensors. Materials today. $2005 ; 8: 30-6$.

17. Braun T, Barwich V, Ghatkesar MK, Bredekamp AH, Gerber C, Hegner M, et al. Micromechanical mass sensors for biomolecular detection in a physiological environment. Physical Review E. 2005;72(3):031907.

18. Ghatkesar MK, Barwich V, Braun T, Ramseyer J, Gerber C, Hegner $\mathrm{M}$, et al. Higher modes of vibration increase mass sensitivity in nanomechanical microcantilevers. Nanotechnology. 2007;18:445502. doi: 10.1088/0957-4484/18/44/445502. PubMed PMID: 000250138500011.

19. Ghatkesar MK, Braun T, Barwich V, Ramseyer J, Gerber C, Hegner M, et al. Resonating modes of vibrating microcantilevers in liquid. Appl Phys Lett. 2008;92:3106-9. doi: 10.1063/1.2838295. PubMed PMID: 2008ApPhL..92d3106G.

20. Padovani F, Duffy J, Hegner M. Microrheological Coagulation Assay Exploiting Micromechanical Resonators. Analytical Chemistry. 2016;89(1):751-8.

21. Padovani F, Duffy J, Hegner M. Nanomechanical clinical coagulation diagnostics and monitoring of therapies. Nanoscale. 2017;9(45):17939-47.

22. Walther M, Fleming PM, Padovani F, Hegner M. An optimized measurement chamber for cantilever array measurements in liquid incorporating an automated sample handling system. EPJ Techniques and Instrumentation. 2015;2(1):7.

23. Ziegler C. Cantilever-based biosensors. Analytical and Bioanalytical Chemistry. 2004;379(7):946-59. doi: 10.1007/ s00216-004-2694-y.

24. Duffy J, Padovani F, Brunetti G, Noy P, Certa U, Hegner M. Towards personalised rapid label free miRNA detection for cancer and liver injury diagnostics in cell lysates and blood based samples. Nanoscale. 2018;10:12797-804.

25. Mishra R, Hegner M. Effect of non-specific species competition from total RNA on the static mode hybridization response of nanomechanical assays of oligonucleotides. Nanotechnology. 2014;25(22):225501.

26. Zhang J, Lang HP, Huber F, Bietsch A, Grange W, Certa $\mathrm{U}$, et al. Rapid and label-free nanomechanical detection of biomarker transcripts in human RNA. Nature Nanotechnology. 2006;1(3):214-20. PubMed PMID: WOS:000243902800018.

27. Backmann N, Zahnd C, Huber F, Bietsch A, Plückthun A, Lang H-P, et al. A label-free immunosensor array using single-chain antibody fragments. Proceedings of the National Academy of Sciences of the United States of America. 2005;102(41):14587-92.

28. Huber F, Hegner M, Gerber C, Guntherodt HJ, Lang HP. Label free analysis of transcription factors using microcantilever arrays. Biosensors \& Bioelectronics. 2006;21(8):1599-605. PubMed PMID: ISI:000235492900025.

29. Gfeller KY, Nugaeva N, Hegner M. Rapid biosensor for detection of antibiotic-selective growth of Escherichia coli. Appl Environ Microbiol. 2005;71:2626-31. doi: 10.1128/AEM.71.5.26262631.2005. PubMed PMID: 000229105300054.

30. Maloney N, Lukacs G, Jensen J, Hegner M. Nanomechanical Sensors for Single Microbial Cell Growth Monitoring. Nanoscale. 2014;6:8242-9. 